\title{
Chaos Driven Evolutionary Algorithm: a Novel Approach for Optimization
}

\author{
Roman Senkerik, Michal Pluhacek, Zuzana Kominkova Oplatkova \\ Faculty of Applied Informatics \\ Tomas Bata University in Zlin \\ T.G. Masaryka 5555, 76001 Zlin, Czech Republic \\ \{senkerik , pluhacek, kominkovaoplatkova \}@fai.utb.cz
}

Received: March 24, 2021. Revised: November 26, 2021. Accepted: December 22, 2021. Published: January 7, 2022.

\begin{abstract}
This research deals with the initial investigations on the concept of a chaos-driven evolutionary algorithm Differential evolution. This paper is aimed at the embedding of simple two-dimensional chaotic system, which is Lozi map, in the form of chaos pseudo random number generator for Differential Evolution. The chaotic system of interest is the discrete dissipative system. Repeated simulations were performed on standard benchmark Schwefel's test function in higher dimensions. Finally, the obtained results are compared with canonical Differential Evolution.
\end{abstract}

Keywords-Evolutionary algorithms; Differential evolution; Chaos; Lozi map

\section{INTRODUCTION}

These days the methods based on soft computing such as neural networks, evolutionary algorithms, fuzzy logic, and genetic programming are known as powerful tool for almost any difficult and complex optimization problem. Ant Colony (ACO), Genetic Algorithms (GA), Differential Evolution (DE), Particle Swarm Optimization (PSO) and Self Organizing Migration Algorithm (SOMA) are some of the most potent heuristics available.

Recent studies have shown that Differential Evolution [1] has been used for a number of optimization tasks, [2], [3] has explored DE for combinatorial problems, [4] has hybridized DE whereas [5] - [7] has developed self-adaptive DE variants.

This paper is aimed at investigating the chaos driven DE. Although a several of papers have been recently focused on the connection of DE and chaotic dynamics either in the form of hybridizing of DE with chaotic searching algorithm [8] or in the form of chaotic mutation factor and dynamically changing weighting and crossover factor in self-adaptive chaos differential evolution (SACDE) [9], the focus of this paper is the embedding of chaotic systems in the form of chaos number generator for DE and its comparison with the canonical DE.

This research is an extension and continuation of the previous successful initial application based experiment with chaos driven DE [10] - [12] with simple test functions in low

This work was supported by European Regional Development Fund under the project CEBIA-Tech No. CZ.1.05/2.1.00/03.0089; and by Internal Grant Agency of Tomas Bata University under the project No. IGA/FAI/2013/012. dimensions.

The primary aim of this work is not to develop a new type of pseudo random number generator, which should pass many statistical tests, but to try to use and test the implementation of natural chaotic dynamics into evolutionary algorithm as a chaotic pseudo random number generator.

The chaotic system of interest is the simple discrete dissipative chaotic system. The two-dimensional Lozi map was selected as the chaos pseudo random number generators for DE based on the successful results obtained with DE [10] or PSO algorithm [13].

Firstly, Differential Evolution is explained. The next sections are focused on the used chaotic systems and test function. Results and conclusion follow afterwards.

\section{DiFFERENTIAL EVOLUTION}

DE is a population-based optimization method that works on real-number-coded individuals [14]. For each individual $\vec{x}_{i, G}$ in the current generation G, DE generates a new trial individual $\vec{x}_{i, G}^{\prime}$ by adding the weighted difference between two randomly selected individuals $\vec{x}_{r 1, G}$ and $\vec{x}_{r 2, G}$ to a randomly selected third individual $\vec{x}_{r 3, G}$. The resulting individual $\vec{x}_{i, G}^{\prime}$ is crossed-over with the original individual $\vec{x}_{i, G}$. The fitness of the resulting individual, referred to as a perturbed vector $\vec{u}_{i, G+1}$, is then compared with the fitness of $\vec{x}_{i, G}$. If the fitness of $\vec{u}_{i, G+1}$ is greater than the fitness of $\vec{x}_{i, G}$, then $\vec{x}_{i, G}$ is replaced with $\vec{u}_{i, G+1}$; otherwise, $\vec{x}_{i, G}$ remains in the population as $\vec{x}_{i, G+1}$. DE is quite robust, fast, and effective, with global optimization ability. It does not require the objective function to be differentiable, and it works well even with noisy and time-dependent objective functions. Description of the used DERand1Bin strategy (both for Chaos DE and Canonical DE) is presented in (1). Please refer to [14] - [17] for the detailed complete description of all other strategies.

$$
u_{j, i, G+1}=x_{j, r 1, G}+F \cdot\left(x_{j, r 2, G}-x_{j, r 3, G}\right)
$$




\section{CHAOTIC LOZI MAP}

This section contains the description of discrete dissipative chaotic map used as the chaotic pseudo random generators for DE. In this research, direct output iterations of the chaotic maps were used for the generation of real numbers in the process of crossover based on the user defined $C R$ value and for the generation of the integer values used for selection of individuals. The initial concept of embedding chaotic dynamics into the evolutionary algorithms is given in [18].

Lozi map is the selected example of chaotic system, which represents the simple discrete two-dimensional chaotic map. The $x, y$ plot of the Lozi map is depicted in Fig. 1. The map equations are given in (2). The parameters are: $a=1.7$ and $b=0.5$ as suggested in [19]. The chaotic behavior of the Lozi map, represented by the examples of direct output iterations are depicted in Fig. 2 (line-plot) and Fig. 3 (point-plot).

$$
\begin{aligned}
& X_{n+1}=1-a\left|X_{n}\right|+b Y_{n} \\
& Y_{n+1}=X_{n}
\end{aligned}
$$

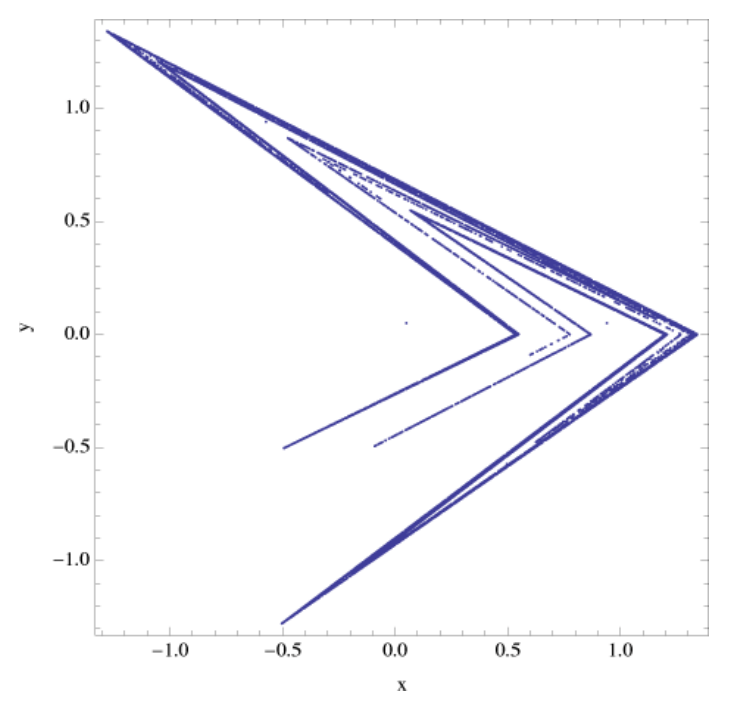

Fig. 1. $x, y$ plot of the Lozi map

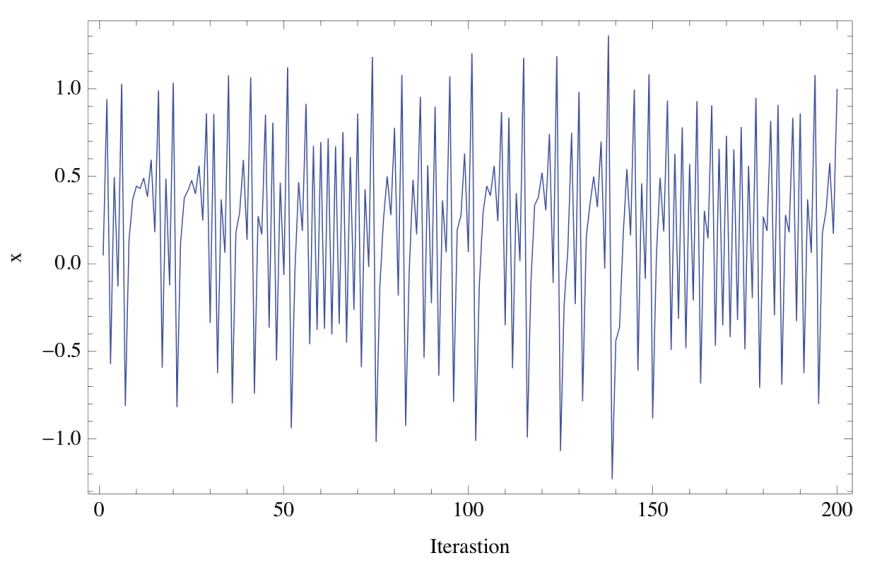

Fig. 2. Iterations of the Lozi map (variable $x$ - line-plot)

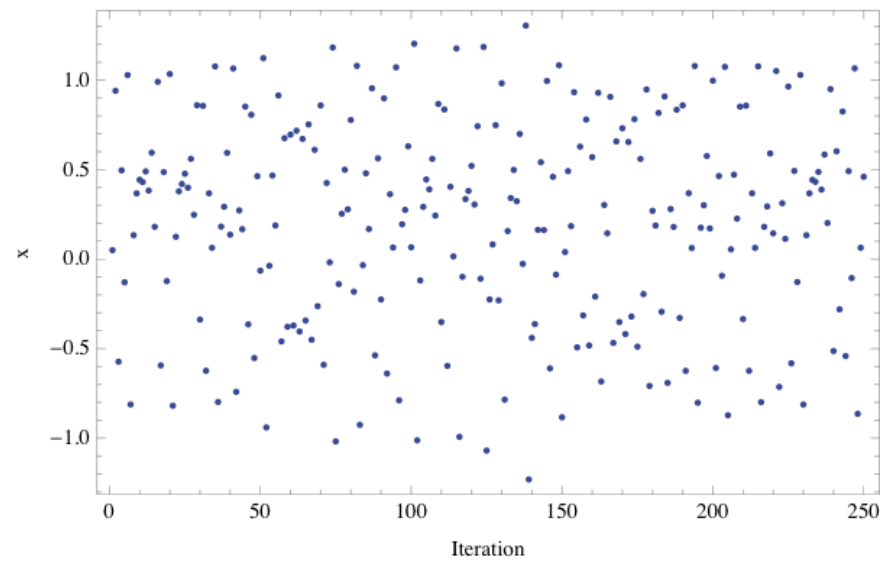

Fig. 3. Iterations of the Lozi map (variable $x$ - point-plot)

The illustrative histogram of the distribution of real numbers transferred into the range $<0$ - $1>$ generated by means of chaotic Lozi map is in Fig. 4

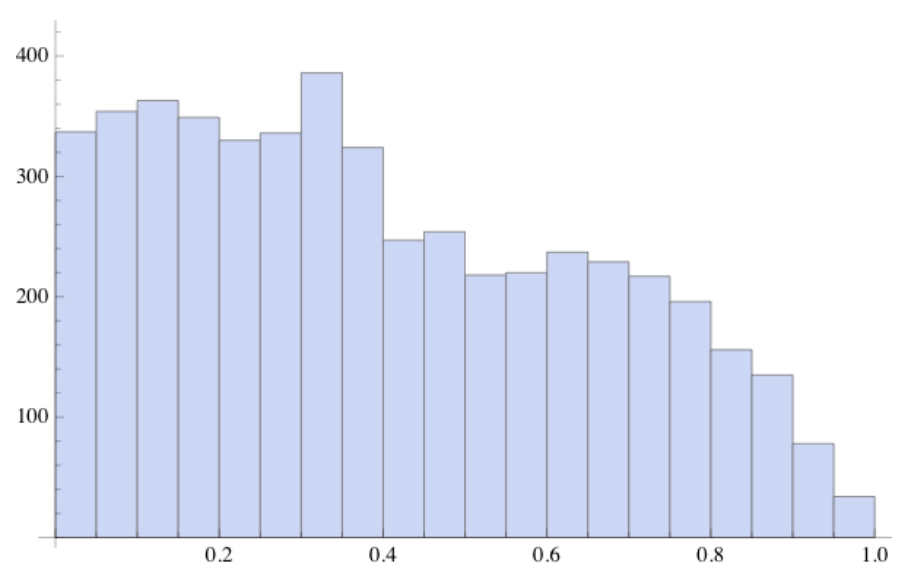

Fig. 4. Histogram of the distribution of real numbers transferred into the range $<0-1>$ generated by means of the chaotic Lozi map - 5000 samples

\section{BENCHMARK FUNCTION}

For the purpose of evolutionary algorithms performance comparison within this initial research, the Schwefel's test function (3) was selected. The 3D diagram for $D=2$ is depicted in Fig. 5, and the 2D diagram for $D=1$ is depicted in Fig. 6.

$$
f(x)=\sum_{i=1}^{D}-x_{i} \sin \left(\sqrt{\left|x_{i}\right|}\right)
$$

Function minimum:

Position for $\mathrm{E}_{\mathrm{n}}:\left(x_{1}, x_{2} \ldots x_{\mathrm{n}}\right)=(420.969,420.969, \ldots, 420.969)$

Value for $\mathrm{E}_{\mathrm{n}}: y=-418.983 \cdot$ Dimension 


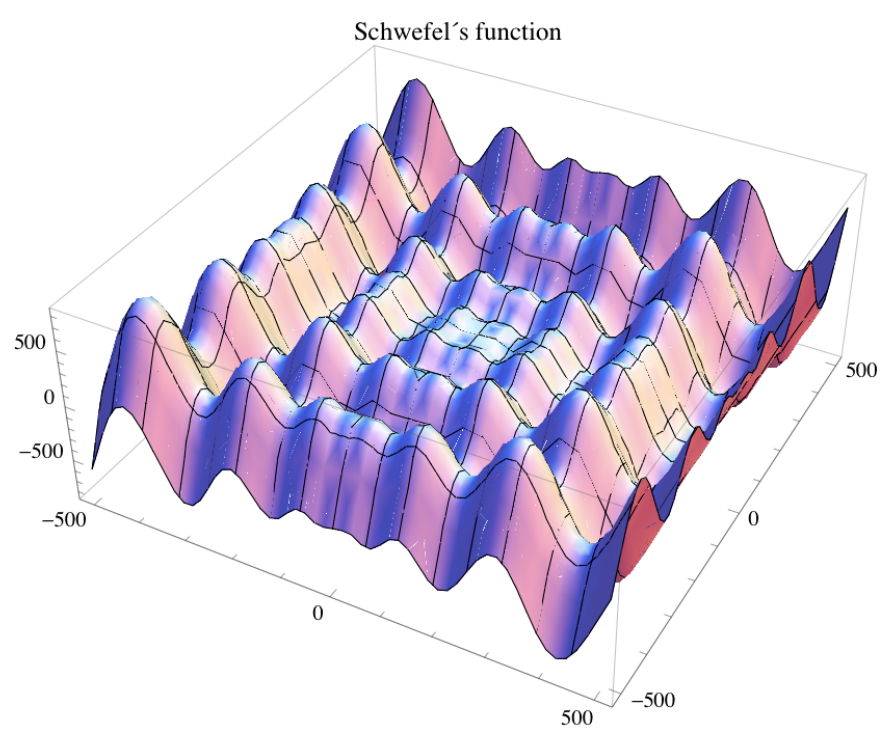

Fig. 5. 3D plot of Schwefel's function

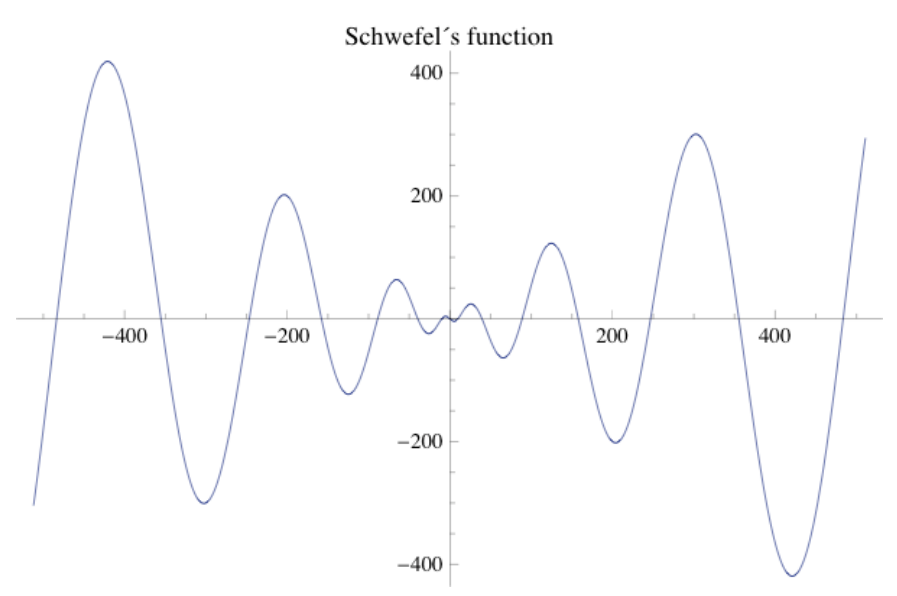

Fig. 6. 2D plot of Schwefel's function

\section{RESUlTS}

The novelty of this approach represents the utilization of discrete chaotic map as a pseudo random number generator for DE. In this paper, the canonical DE strategy DERand1Bin and the Chaos DERand1Bin strategy driven by Lozi map (ChaosDE) were used. The parameter settings for both canonical DE and ChaosDE were obtained analytically based on numerous experiments and simulations (see Table 1). Experiments were performed in an environment of Wolfram Mathematica, canonical DE therefore used the built-in Mathematica software pseudo random number generator. All experiments used different initialization, i.e. different initial population was generated in each run of Canonical or Chaos driven DE.

Within this research, one experiment was performed. It utilizes the maximum number of generations fixed at 3000 generations. This allowed the possibility to analyze the progress of DE within a limited number of generations and cost function evaluations.

The results of the experiment are shown in Table 2, which represent the simple statistics for cost function values, e.g. average, median, maximum values, standard deviations and minimum values representing the best individual solution for all 50 repeated runs of canonical DE and ChaosDE.

The main aim of the optimization was to find the global extreme (minimum) of the Schwefel's test function in higher dimensions. For $D=30$, the global minimum has the following value $\mathrm{E}_{\mathrm{n}}: y=-12569.49$.

Table 3 compares the progress of ChaosDe and Canonical DE. The Table 3 contains the average CF values for the generation No. 750, 1500, 2250 and 3000 from all 50 runs.

The bold values within the both Table 2 and Table 3 depict the best obtained results.

TABLE I. PARAMETER SET UP FOR CANONICAL DE AND CHAOSDE

\begin{tabular}{|l|c|}
\hline \multicolumn{1}{|c|}{ DE Parameter } & Value \\
\hline Popsize & 75 \\
\hline $\mathrm{F}$ & 0.8 \\
\hline $\mathrm{Cr}$ & 0.8 \\
\hline Dimensions & 30 \\
\hline Generations & $100 \cdot \mathrm{D}=3000$ \\
\hline Max Cost Function Evaluations (CFE) & 225000 \\
\hline
\end{tabular}

TABLE II. SIMPLE RESUlts STATISTICS

\begin{tabular}{|l|c|c|}
\hline CF statistical parameter & Canonical DE & ChaosDE \\
\hline Average CF & -5944.01 & $\mathbf{- 1 0 8 8 3 . 5}$ \\
\hline Median CF & -5961.63 & $\mathbf{- 1 0 9 6 6 . 5}$ \\
\hline Max. CF & -5412.69 & $\mathbf{- 7 6 0 9 . 9 4}$ \\
\hline Min. CF & -7045.82 & $\mathbf{- 1 2 4 2 7 . 6}$ \\
\hline Std. Dev. & $\mathbf{2 6 2 . 2 3 2}$ & 996.605 \\
\hline
\end{tabular}

TABLE III. COMPARISON OF Progress TOWARdS THE Minimum

\begin{tabular}{|l|c|c|c|c|}
\hline DE Version & $\begin{array}{c}\text { Avg. CF value } \\
\text { for Gen. No. } \\
\mathbf{7 5 0}\end{array}$ & $\begin{array}{c}\text { Avg. CF value } \\
\text { for Gen. No. } \\
\mathbf{1 5 0 0}\end{array}$ & $\begin{array}{c}\text { Avg. CF value } \\
\text { for Gen. No. } \\
\mathbf{2 2 5 0}\end{array}$ & $\begin{array}{c}\text { Avg. CF value } \\
\text { for Gen. No. } \\
\mathbf{3 0 0 0}\end{array}$ \\
\hline Canonical DE & -5341.68 & -5591.21 & -5831.25 & -5944.01 \\
\hline ChaosDE & $\mathbf{- 5 5 1 9 . 1 1}$ & $\mathbf{- 7 6 2 5 . 5 9}$ & $\mathbf{- 9 5 0 1 . 4 6}$ & $\mathbf{- 1 0 8 8 3 . 5}$ \\
\hline
\end{tabular}

Obtained numerical results and graphical comparisons in Fig. 7 - 10 support the claim that Chaos DE driven by Lozi map has given the best overall results. The graphical comparison of the time evolution of $\mathrm{CF}$ values for the best individual solutions (the solution with the minimal final cost function value) for Chaos $\mathrm{DE}$ with Lozi map and canonical DERand1Bin strategy is depicted in Fig. 7, whereas Fig. 8 represents the comparison of the time evolution of average $\mathrm{CF}$ values from all 50 runs. Fig. 9 shows the time evolution of $\mathrm{CF}$ values for the best progressive individual solutions. These individual solutions represent the ones with the lowest sum of the $\mathrm{CF}$ values with the step of 20 generations, i.e. with the best progress towards the global optimum. Finally the Fig. 10 
confirms the robustness of Chaos DE driven by chaotic Lozi map in finding the best solutions for all 50 runs.

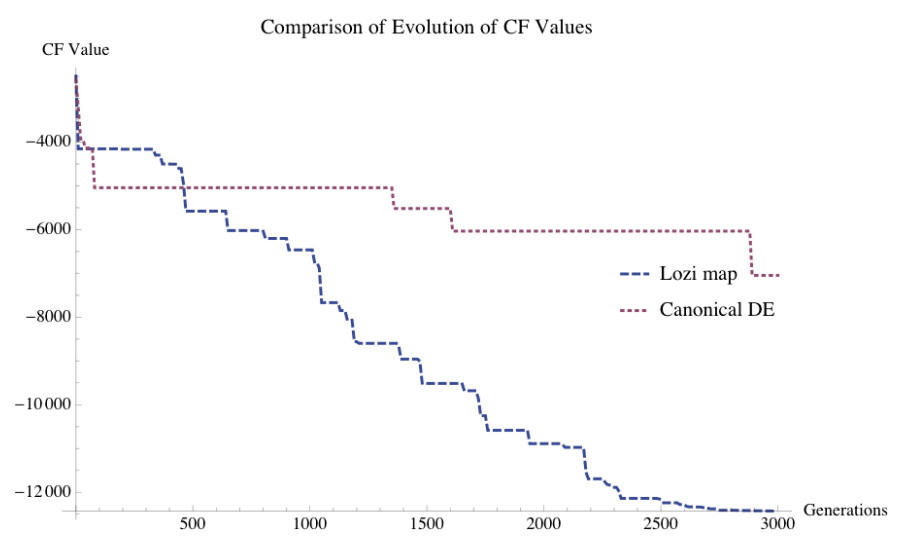

Fig. 7. Comparison of the time evolution of $\mathrm{CF}$ values for the best individual solutions, i.e. the solutions with the minimal final cost function value

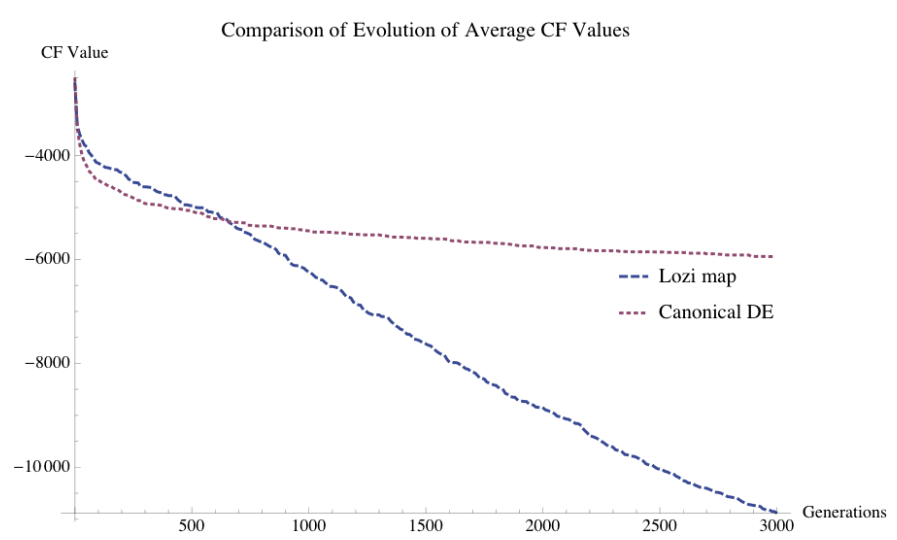

Fig. 8. Comparison of the time evolution of average CF values for all 50 runs of ChaosDE and Canonical DE

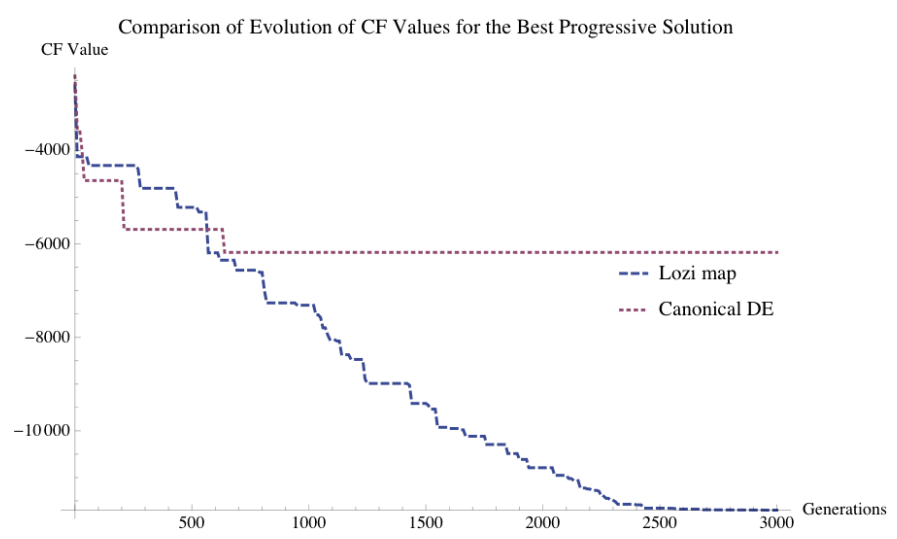

Fig. 9. Comparison of time evolution of $\mathrm{CF}$ values for the best progressive individual solutions, i.e. solutions with the lowest sum of the CF values with the step of 20 generations

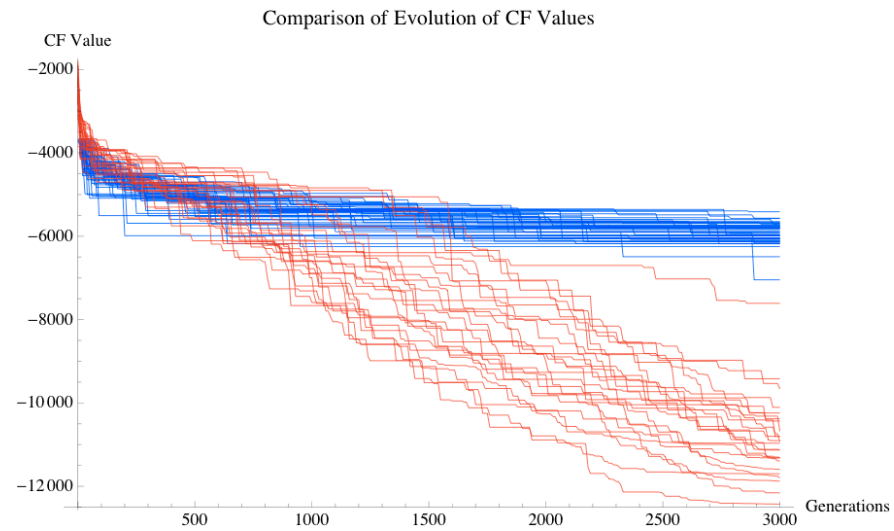

Fig. 10. Comparison of the time evolution of $\mathrm{CF}$ values for all 50 runs of canonical DE (blue) and ChaosDE (red)

\section{CONCLUSIONS}

In this paper, chaos driven DERand1Bin strategy was tested and compared with canonical DERand1Bin strategy. Based on obtained results, it may be claimed, that the developed ChaosDE driven by means of the chaotic Lozi map gives considerably better results than other compared heuristics.

Since this was an initial study, future plans include experiments with benchmark functions in higher dimensions, testing of different chaotic systems and obtaining a large number of results to perform statistical tests.

Furthermore chaotic systems have additional parameters, which can by tuned. This issue opens up the possibility of examining the impact of these parameters to generation of random numbers, and thus influence on the results obtained using differential evolution.

\section{REFERENCES}

[1] K. Price," An Introduction to Differential Evolution", In: New Ideas in Optimization, (D. Corne, M. Dorigo and F. Glover, Eds.), McGraw-Hill, London, UK, pp. 79-108, 1999, ISBN 007-709506-5, 1999.

[2] M. F. Tasgetiren, P. N. Suganthan and Q. K. Pan, "An Ensemble of Discrete Differential Evolution Algorithms for Solving the Generalized Traveling Salesman Problem", Applied Mathematics and Computation, Vol. 215, Issue 9, p. 3356 - 3368, 2010.

[3] G. Onwubolu and D. Davendra (Eds), "Differential Evolution: A handbook for Permutation-based Combinatorial Optimization", Springer, Germany, 2009

[4] S. Das, A. Konar, U. K. Chakraborty and A. Abraham, "Differential evolution with a neighborhood based mutation operator: a comparative study", IEEE Transactions on Evolutionary Computations, vol. 13, no. 3, 2009, pp. 526-553.

[5] A. K. Qin, V. L. Huang and P. N. Suganthan, "Differential evolution algorithm with strategy adaptation for global numerical optimization", IEEE Transactions on Evolutionary Computations, vol. 13, no. 2, 2009, pp. 398-417.

[6] J. Zhang and A. C. Sanderson, "JADE: Self-adaptive differential evolution with fast and reliable convergence performance", In Proceedings of IEEE Congress on Evolutionary. Computation., Singapore, 2007, pp. 2251-2258. 
[7] J. Zhang and A. C, Sanderson, "Self-adaptive multiobjective differential evolution with direction information provided by archived inferior solutions", In Proceedings of IEEE World Congress on Evolutionary. Computation Hong Kong, 2008, p. $2801-2810$.

[8] W. Liang, L. Zhang and M. Wang, "The Chaos Differential Evolution Optimization Algorithm and its Application to Support Vector Regression Machine", Journal Of Software, Vol. 6, No. 7, pp. 12971304, 2011.

[9] G. Zhenyu, Ch. Bo, Z. Min and C. Binggang, "Self-Adaptive Chaos Differential Evolution", Lecture Notes in Computer Science, 2006, Volume 4221, pp. 972-975, 2006.

[10] D. Davendra, I. Zelinka and R. Senkerik, "Chaos driven evolutionary algorithms for the task of PID control", Computers \& Mathematics with Applications, Vol. 60, Issue 4, 2010, pp 1088-1104, ISSN 0898-1221.

[11] R. Senkerik, D. Davendra, I. Zelinka, M. Pluhacek and Z. Oplatkova, "An Investigation on the Chaos Driven Differential Evolution: An Initial Study", In: Proceedings of 5th International Conference on Bioinspired Optimization Methods and Their Applications, BIOMA 2012, pp. 185 194, 2012, ISBN 978-961-264-043-9.

[12] R. Senkerik, D. Davendra, I. Zelinka, M. Pluhacek and Z. Oplatkova, "An Investigation On The Differential Evolution Driven By Selected Discrete Chaotic Systems", In: Proceedings of the 18th International Conference on Soft Computing - MENDEL 2012, 2012, pp. 157 - 162, 2012, ISBN 978-80-214-4540-6.
[13] M. Pluhacek, R. Senkerik, D. Davendra, Z. Kominkova Oplatkova and I. Zelinka, "On the behavior and performance of chaos driven PSO algorithm with inertia weight", Computers \& Mathematics with Applications, 2013, (article in press), ISSN 0898-1221, DOI 10.1016/j.camwa.2013.01.016.

[14] R. Storn, K. Price, "Differential evolution - a simple and efficient heuristic for global optimization over continuous spaces", Journal of Global Optimization, vol. 11, 1997, pp. 341-359.

[15] K. Price, "An Introduction to Differential Evolution", In: New Ideas in Optimization, (D. Corne, M. Dorigo and F. Glover, Eds.), pp. 79-108, McGraw-Hill, London, UK, ISBN 007-709506-5, 1999.

[16] K. Price and R. Storn, "Differential evolution homepage" (2001) [Online]. Available: http://www.icsi.berkeley.edu/ storn/code.html

[17] K. Price, R. Storn and J. Lampinen, "Differential Evolution - A Practical Approach to Global Optimization", 2005, Springer, ISBN: 3-540-209506.

[18] R. Caponetto, L. Fortuna, S. Fazzino and M. Xibilia, "Chaotic sequences to improve the performance of evolutionary algorithms", IEEE Trans. Evol. Comput, vol. 7:3. 2003, pp. 289-304.

[19] Sprott J. C., "Chaos and Time-Series Analysis", Oxford University Press, 2003.

\section{Creative Commons Attribution License 4.0 (Attribution 4.0 International, CC BY 4.0)}

This article is published under the terms of the Creative Commons Attribution License 4.0 https://creativecommons.org/licenses/by/4.0/deed.en US 